\title{
Safety and efficacy of a Nav1.7 selective sodium channel blocker in patients with trigeminal neuralgia: a double-blind, placebo-controlled, randomised withdrawal phase 2 a trial
}

\author{
Zakrzewska, Joanna M ; Palmer, Joanne ; Morisset, Valerie ; Giblin, Gerard M ; Obermann, Mark ; \\ Ettlin, Dominik A ; Cruccu, Giorgio ; Bendtsen, Lars ; Estacion, Mark ; Derjean, Dominique ; \\ Waxman, Stephen G ; Layton, Gary ; Gunn, Kevin ; Tate, Simon
}

\begin{abstract}
Background: Current standard of care for trigeminal neuralgia is treatment with the sodium channel blockers carbamazepine and oxcarbazepine, which although effective are associated with poor tolerability and the need for titration. BIIB074, a Nav1.7-selective, state-dependent sodium-channel blocker, can be administered at therapeutic doses without titration, and has shown good tolerability in healthy individuals in phase 1 studies. We therefore assessed the safety and efficacy of BIIB074 in patients with trigeminal neuralgia in a phase 2a study. Methods: We did a double-blind, multicentre, placebocontrolled, randomised withdrawal phase 2a trial in 25 secondary care centres in Denmark, Estonia, France, Germany, Italy, Latvia, Lithuania, Romania, South Africa, Spain, Switzerland, and the UK. After a 7-day run-in phase, eligible patients aged 18-80 years with confirmed trigeminal neuralgia received openlabel, BIIB074 $150 \mathrm{mg}$ three times per day, orally, for 21 days. Patients who met at least one response criteria were then randomly assigned (1:1) to BIIB074 or placebo for up to 28 days in a double-blind phase. We used an interactive web response system to assign patients with a computer-generated schedule, with stratification (presence or absence of existing pain medication). Patients, clinicians, and assessors were masked to treatment allocation. The primary endpoint was the difference between groups in the number of patients classified as treatment failure during the double blind phase assessed in the modified intentionto-treat population. We assessed safety in all patients who received one or more doses of BIIB074. This study is registered with ClinicalTrials.gov (NCT01540630) and EudraCT (2010-023963-16). Findings: The first patient was enrolled on April 23, 2012, and the last patient completed the study on February 26, 2014. We enrolled 67 patients into the open-label phase; 44 completed open-label treatment, and 29 were randomly assigned to double-blind treatment (15 to BIIB074 and 14 to placebo). During the double-blind phase, five (33\%) patients assigned to BIIB074 versus nine (64\%) assigned to placebo were classified as treatment failures $(\mathrm{p}=0 \cdot 0974)$. BIIB074 was well tolerated, with similar adverse events in the double-blind phase to placebo. Headache was the most common adverse event with BIIB074 in the open-label phase (in 13 [19\%] of 67 patients), followed by dizziness (in six [9\%] patients). In the double-blind phase, headache, pyrexia, nasopharyngitis, sleep disorder, and tremor were the most frequent adverse events in patients assigned to BIIB074 (in one [7\%] of 15 patients for each event), and headache, dizziness, diarrhoea, and vomiting were the most frequent adverse events in patients assigned to placebo (in one [7\%] of 14 patients for each event). No severe or serious adverse events were reported in the BIIB074 group during the double-blind phase. One patient assigned to placebo reported intestinal adhesions with obstruction as a severe and serious adverse event, which was considered as unrelated to study medication. Interpretation: The primary endpoint of treatment failure was not significantly lower in the BIIB074 group than in the placebo group. However, our findings provide a basis for continued investigation of BIIB074 in patients with trigeminal neuralgia in future clinical trials.
\end{abstract}

DOI: https://doi.org/10.1016/S1474-4422(17)30005-4 
Posted at the Zurich Open Repository and Archive, University of Zurich

ZORA URL: https://doi.org/10.5167/uzh-135668

Journal Article

Accepted Version

Originally published at:

Zakrzewska, Joanna M; Palmer, Joanne; Morisset, Valerie; Giblin, Gerard M; Obermann, Mark; Ettlin, Dominik A; Cruccu, Giorgio; Bendtsen, Lars; Estacion, Mark; Derjean, Dominique; Waxman, Stephen G; Layton, Gary; Gunn, Kevin; Tate, Simon (2017). Safety and efficacy of a Nav1.7 selective sodium channel blocker in patients with trigeminal neuralgia: a double-blind, placebo-controlled, randomised withdrawal phase 2a trial. Lancet Neurology, 16(4):291-300.

DOI: https://doi.org/10.1016/S1474-4422(17)30005-4 
TN Phase 2

\section{Safety and efficacy of BIIB074, a Nav1.7-selective sodium channel blocker, in} trigeminal neuralgia: a double-blind, placebo-controlled, randomised withdrawal phase 2 trial

Prof Joanna M Zakrzewska, MD¹, Joanne Palmer, $\mathrm{PhD}^{2}$, Valerie Morisset, $\mathrm{PhD}^{2}$, Gerard MP Giblin, $\mathrm{PhD}^{2}$, Mark Obermann, $\mathrm{MD}^{3}$, Dominik A Ettlin, MD, DMD ${ }^{4}$, Prof Giorgio Cruccu MD ${ }^{5}$, Lars Bendtsen, MD, $\mathrm{PhD}^{6}$, Mark Estacion, $\mathrm{PhD}^{7}$, Dominique Derjean, $\mathrm{PhD},{ }^{2}$ Prof Stephen $\mathrm{G}$ Waxman, $\mathrm{MD}, \mathrm{PhD}^{7}$, Gary Layton, $\mathrm{MSc}^{8}$, Kevin Gunn, $\mathrm{MD}^{2^{*}}$ and Simon Tate, $\mathrm{PhD}^{2}$, on behalf of the Study Investigators

${ }^{1}$ Facial Pain Unit, Division of Diagnostic, Surgical and Medical Sciences, Eastman Dental Hospital, University College London Hospitals NHS Foundation Trust / University College London, London, UK; ${ }^{2}$ Convergence Pharmaceuticals Ltd, a Biogen company, Cambridge, UK; ${ }^{3}$ Department of Neurology and German Headache Center, University of DuisburgEssen, Essen, Germany, and Center for Neurology, Asklepios Hospitals Schildautal, Seesen, Germany; ${ }^{4}$ Interdisciplinary Orofacial Pain Unit, Clinic KFS-KAB, Center of Dental Medicine, University of Zurich, Zurich, Switzerland; ${ }^{5}$ Department of Neurology and Psychiatry, Sapienza University, Roma, Italy; ${ }^{6}$ Danish Headache Centre, Department of Neurology, Rigshospitalet - Glostrup, University of Copenhagen, Nordre Ringvej 67, 2600 Glostrup, Denmark; ${ }^{7}$ Department of Neurology and Center for Neuroscience \& Regeneration Research, Yale University School of Medicine, and Veterans Affairs Medical Center, West Haven, CT, USA; ${ }^{8}$ ParamStat Ltd, Ash, UK *Kevin Gunn was an employee of Convergence Pharmaceuticals Ltd at the time this study was conducted, and is currently retired.

Corresponding author: Joanna M Zakrzewska, Eastman Dental Hospital, UCLH NHS Foundation Trust, 256 Gray's Inn Road, London WC1X 8LD, UK. Tel: + 44 (0) 20345 61195; email: J.Zakrzewska@ucl.ac.uk 
TN Phase 2

\section{Summary}

Word count (Max=300): 358

Background: Current standard of care for trigeminal neuralgia is treatment with the sodium channel blockers carbamazepine and oxcarbazepine. Unlike these drugs, BIIB074 (CNV1014802), a novel Nav1.7-selective, state-dependent sodium channel blocker, can be administered at immediate therapeutic doses and has shown good tolerability in healthy individuals in phase 1 studies. We therefore assessed the safety and efficacy of BIIB074 in trigeminal neuralgia.

Methods: This phase $2 \mathrm{a}$, double-blind withdrawal trial was conducted in 25 secondary care centres in the UK, Germany, Denmark, Italy, Switzerland, South Africa, France, Spain, Estonia, Latvia, Lithuania, and Romania. After a 7-day run-in, patients aged 18-80 years with classical trigeminal neuralgia received open-label BIIB074 150-mg three times a day for 21 days. Patients meeting response criteria at the end of the open-label phase were randomised to BIIB074 or placebo in a 28-day double-blind phase. Patients, clinicians, and assessors were masked to treatment allocation, with tablets for study drug and placebo identical in appearance and packaging. Primary endpoint was the proportion meeting treatment failure criteria with BIIB074 versus placebo during the double-blind phase in the modified intention-to-treat population. This study is registered with ClinicalTrials.gov (NCT01540630) and EudraCT (2010-023963-16).

Findings: The first patient was enrolled on April 23, 2012, and the last patient completed the study on Feb 26, 2014. Sixty-seven patients entered the open-label phase; 44 completed open-label treatment, and 29 were randomised and received double-blind treatment $(n=15$ BIIB074, $n=14$ placebo). During the double-blind phase, five (33\%) BIIB074 versus nine (64\%) placebo patients were classified as treatment failures (one-sided $p=0.0974$ ). BIIB074 was well-tolerated, with double-blind phase adverse events similar to placebo. There were no severe or serious AEs reported in the BIIB074 group during the double-blind phase. One patient on placebo (7\%) reported a severe $A E$ which also reported as a serious adverse event (intestinal adhesions with obstruction), considered as unrelated to study medication. 
TN Phase 2

Interpretation: The primary endpoint of this trial did not reach statistical significance; however, fewer patients treated with BIIB074 were classified as treatment failures compared with placebo. We believe that our findings provide a basis for continued investigation of BIIB074 in trigeminal neuralgia.

Funding: Convergence Pharmaceuticals Ltd., a Biogen company. 
TN Phase 2

\section{Panel: Research in context}

\section{Evidence before this study}

Based on a search of PubMed (search terms 'trigeminal neuralgia', June 1 2005-June 16 2015, English-language) and the authors' own publications, we identified the following comprehensive systematic reviews of the evidence base for drug treatments in trigeminal neuralgia: Cochrane reviews by Wiffen et al (2014), Zhang et al (2013) and Wiffen et al (2013), a Clinical Evidence review (Zakrzewska and Linskey 2014), and international clinical guidelines (Cruccu 2008). The individual publications report their search strategy. Overall, this literature highlights a lack of high quality clinical trials in trigeminal neuralgia. Current standard of care and guideline-recommended first-line treatment is with the sodium channel blockers carbamazepine or oxcarbazepine, but even for these drugs the evidence base is weak ('third-tier' evidence only for carbamazepine and no eligible studies for oxcarbazepine, per Cochrane reviews). Further, these treatments are limited by poor tolerability, need for well-managed titration, and potential for pharmacological interactions.

\section{Added value of this study}

Few advances have been made in the treatment of trigeminal neuralgia in recent decades and high-quality clinical trials have been lacking. This study was designed to address barriers to the conduct of traditional randomised controlled trials in the trigeminal neuralgia population, by utilizing a randomised withdrawal design that limits the exposure of patients to placebo in the absence of benefit. The results of this study indicate potential for BIIB074, a novel Nav1.7-selective sodium channel blocker, to be both effective and well-tolerated in trigeminal neuralgia patients. Notably, in contrast to carbamazepine/oxcarbazepine, BIIB074 was associated with low rates of cognitive impairment, and can be administered without titration to therapeutic doses.

\section{Implications of all the available evidence}


TN Phase 2

To our knowledge, BIIB074 is unique in being developed with trigeminal neuralgia as the primary indication. If successful, the programme could lead to the first US Food and Drug Administration approval for trigeminal neuralgia since the introduction of carbamazepine in the 1960s. We believe that our results provide a basis for the continued investigation of BIIB074 in trigeminal neuralgia. 
TN Phase 2

Body text word count $(\max =4500): 4500$

\section{Introduction}

Trigeminal neuralgia is a rare and at times debilitating orofacial disorder, characterised by unilateral paroxysms of severe pain in the territory innervated by the trigeminal nerve, usually triggered by innocuous stimuli. ${ }^{1}$ Currently, the only US Food and Drug Administration (FDA)-approved treatment for trigeminal neuralgia is the voltage-gated sodium channel (Nav) blocker carbamazepine, which was approved for this disorder in the 1960s.

Carbamazepine and its analogue oxcarbazepine are recommended as first-line treatments for trigeminal neuralgia. ${ }^{2}$ However, although generally considered effective, these treatments are limited by poor tolerability, need for well managed titration, and potential for pharmacological interactions. ${ }^{2-5}$ Other oral drugs (predominantly anticonvulsants) have been investigated in trigeminal neuralgia; however, none has shown a clear benefit. ${ }^{2,6}$ Cochrane reviews have highlighted that overall few high-quality clinical trials in trigeminal neuralgia have been done, and even for carbamazepine, the evidence base is weak. ${ }^{3,6,7} \mathrm{~A}$ recent systematic review indicated that botulinum toxin is effective in trigeminal neuralgia; however, randomised controlled trials were not identified that evaluated duration of effect beyond 3 months. ${ }^{9}$

Nav1.7 is a sodium channel that is preferentially expressed in peripheral neurons, including trigeminal neurons, and results from functional and genetic studies suggest a link between Nav1.7 and pain signalling in humans. ${ }^{10-12}$ Loss-of-function mutations in Nav1.7 are associated with insensitivity to pain in individuals who are apparently cognitively healthy, ${ }^{13}$ suggesting Nav1.7 as a potential target for pain. Efficacy of the Nav blockers carbamazepine (known to block Nav1. $7^{14}$ ) and oxcarbazepine lends further support for Nav1.7 as a target in trigeminal neuralgia.

BIIB074 (CNV1014802) is a novel Nav1.7-selective, state-dependent sodium channel blocker $^{15,16}$ under investigation for the treatment of trigeminal neuralgia. Electrophysiological studies have shown that BIIB074 preferentially inhibits higher frequencies of firing ${ }^{17}$ such as would be expected in the paroxysms of pain in trigeminal neuralgia. ${ }^{18,19}$ Results from phase 
TN Phase 2

1 studies suggest that BIIB074 has good tolerability in healthy individuals and can be administered at therapeutic doses without lengthy titration (unpublished). Thus, if proven efficacious, BIIB074 could fill an unmet need for a trigeminal neuralgia treatment that is both effective and well tolerated, particularly because many patients opt to remain on medications rather than undergo neurosurgical procedures. ${ }^{4}$

We assessed the safety and efficacy of BIIB074 in classical trigeminal neuralgia in a phase 2 a withdrawal trial. ${ }^{20}$

\section{Methods}

\section{Study design and patients}

This trial was a phase $2 a$, placebo-controlled, multicentre, double-blind, randomised withdrawal study (figure S1). The full study design has been published previously, prior to completion of the study. ${ }^{20}$

The study was conducted in 25 international secondary care centres with a special interest in headache and facial pain (across the UK, Germany, Denmark, Italy, Switzerland, South Africa, France, Spain, Estonia, Latvia, Lithuania, and Romania). Patients were identified from databases held at the study centres and by advertising through patient support groups, and were recruited directly from specialised headache and facial pain clinics. All patients provided written informed consent. The study protocol, patient information, informed consent forms, and amendments, were reviewed and approved by relevant independent ethics committees or institutional review boards, and the study was conducted in accordance with the International Conference on Harmonisation principles of Good Clinical Practice and principles of the Declaration of Helsinki.

Adults aged 18-80 years (increased from 70 years in a protocol amendment, MayAugust 2012, detailed in online appendix) meeting criteria for classical trigeminal neuralgia based on the International Classification of Headache Disorders (ICHD) were included in the study. ${ }^{1,20,21}$ Each clinical diagnosis was additionally verified by the trial's data monitoring committee (DMC), and confirmed prior to entry into the treatment phase. Protocol criteria for 
TN Phase 2

classical trigeminal neuralgia were based on the ICHD Second Edition; however, the DMC also considered criteria of the Third Edition published in 2013. ${ }^{1,21}$ Patients had undergone imaging to ensure no secondary cause was present, and were also excluded if there were signs of dental causes, autonomic symptoms, or other neuropathic pain. Following screening, patients were enrolled in a 7-day run-in period, during which the patients recorded number and severity of paroxysms of pain on a paper diary and completed a washout of prohibited medications. To be eligible for inclusion in the open-label phase, patients had to have at least three paroxysms of pain per day, each rated at intensity $\geq 4$ on a pain intensity numerical rating scale (PI-NRS) on at least 4 days during run-in. Further details of the essential criteria for diagnosis of trigeminal neuralgia and other inclusion/exclusion criteria are reported in the appendix and in the published protocol. ${ }^{20}$

Following run-in, eligible patients initially entered a 21-day open-label treatment phase, during which they received open-label BIIB074 $150 \mathrm{mg}$ orally three-times daily (tid) for 21 days with no upward titration. On Day 21 , response criteria were assessed to determine eligibility for a 28-day double-blind phase. Eligibility was determined on the basis of meeting at least one of the following criteria: $\geq 30 \%$ decrease in the number of paroxysms of pain over the last 7 days of open-label compared with 7 -day run-in; $\geq 30 \%$ reduction in severity of paroxysms of pain over the last 7 days of open-label compared with 7-day run-in; or a Patient Global Impression of Change (PGIC) rating of much improved or very much improved.

Patients entering the double-blind phase were randomised to BlIB074 $150 \mathrm{mg}$ tid or placebo. During the double-blind phase, patients meeting criteria for treatment failure were to be withdrawn from treatment. Treatment failure was determined on the basis of meeting at least one of the following criteria: more than three paroxysms in a 7-day period and either a $\geq 50 \%$ increase in the frequency of paroxysms, or a $\geq 50 \%$ increase in the severity of paroxysms, compared to the final 7 days of open-label; PGIC rating of much worse or very much worse (relative to the end of the open-label phase); or the patient discontinued because of 'lack of efficacy' (as defined and reported by the patient or clinician), or due to an 
TN Phase 2

adverse reaction or poor tolerability considered to be related to the study medication.

Following cessation of double-blind treatment, patients returned for a follow-up visit after 7 days to ascertain their level of pain.

Patients were not permitted to use other sodium channel blockers during the study.

BIIB074 is a potent, reversible monoamine oxidase (MAO)-B inhibitor; therefore, medications that could adversely interact with an MAO-B inhibitor (including other MAO inhibitors) were also excluded. Further details of prohibited medications are provided in the appendix. Permitted concomitant medications for the treatment of trigeminal neuralgia had to have been stable for $\geq 3$ weeks prior to the start of open-label treatment and maintained at stable dose.

\section{Randomisation and masking}

Patients were randomised in the double-blind phase (1:1 to BIIB074 or placebo) according to a computer-generated, centralised randomisation schedule. Patients were allocated to treatment through an interactive web response system accessed by the site, and medication was dispensed from a pharmacy that had no input into patient care. The randomisation schedule was stratified by whether or not the patient was taking existing pain medication. Patients, clinicians, and assessors were masked to treatment allocation, with tablets for study drug and placebo identical in appearance and packaging. Masking was assessed by asking patients and clinicians to guess which treatment had been administered at the end of double-blind treatment. The primary outcome was assessed centrally.

\section{Procedures}

During the open-label phase, all patients were required to take one tablet of BIIB074 $150 \mathrm{mg}$ (film-coated, immediate release) orally three-times per day for 21 days. During the doubleblind phase, patients took one tablet of BIIB074 $150 \mathrm{mg}$ or matching placebo orally threetimes per day for up to 28 days. Tablets had to be taken at least 1 hour before or after food and approximately 8 hours apart. 
TN Phase 2

Patients were asked to record the number of spontaneous or evoked paroxysms of pain experienced per 24-hour period, and the severity of each paroxysm, on a paper diary card. Severity of paroxysms was rated by the patients on an 11-point PI-NRS from 0 to 10, where 0 represents "no pain" and 10 "maximum pain imaginable". Patients were also asked to rate pain intensity averaged over the last 24 hours before retiring to bed (average daily pain score), also recorded using the 11-point PI-NRS) (figure S2). Patient Global Impression of Change (PGIC) and Clinician Global Impression of Change (CGIC) scales were used by the patient and clinician, respectively, to record their opinion of the change in overall status according to a 7-point NRS (1 representing "very much improved" and 7 "very much worse"). The PGIC and CGIC were completed at the end of open-label treatment relative to run-in, and at the end of the double-blind phase or premature discontinuation relative to end of the open-label phase. Patients were also asked to complete the Brief Pain Inventory-Facial (BPIF), ${ }^{22}$ at the start and end of open-label treatment, and at the end of double-blind treatment or premature discontinuation. However, data for BPI-F were collected incompletely and are therefore not reported.

Blood samples for pharmacokinetic analyses were collected pre-dose on day 0 , and pre-dose and $2 \mathrm{~h}$ post-dose on days 7, 21, 35, and 49 (or premature discontinuation).

\section{Outcomes}

The primary endpoint was treatment failure during the double-blind phase. Secondary endpoints were the number (mean number per day calculated over the previous week) and severity of paroxysms (mean severity across all paroxysms in the previous week), average daily pain score (mean of average daily pain over the previous week), PGIC and CGIC scores, and BPI-F (not reported), for the open-label and double-blind phases. ${ }^{20}$

Safety was assessed by monitoring of adverse events (AEs), vital signs, electrocardiogram (ECG) parameters, and laboratory safety tests (including clinical chemistry, haematology and urinalysis). Adverse events (either volunteered spontaneously 
TN Phase 2

by patients or in response to general, non-leading questioning by the investigator) were recorded from the start of treatment on Day 0 until the final follow-up visit.

Pharmacokinetic assessments included the measurement of plasma BIIB074 concentrations pre-dose and $2 \mathrm{~h}$ post-dose, with estimation of maximum $\left(\mathrm{C}_{\text {max-ss }}\right)$ and minimum $\left(\mathrm{C}_{\text {min-ss }}\right)$ concentrations and exposure (area under curve 0-24 $\left.\mathrm{h}\left[\mathrm{AUC}_{0-24-\mathrm{ss}}\right]\right)$ at steady state.

\section{Statistical analysis}

Up to 70 patients were to be recruited into the open-label phase in order to randomise 30 into the double-blind phase. Based on assumptions that $20 \%$ of patients on BIIB074 and $67 \%$ on placebo would meet criteria for treatment failure (defined under 'Study design and patient population') during double-blind treatment, with 11 evaluable patients per treatment arm (22 in total) during the double-blind phase, the study had $80 \%$ power to demonstrate a statistically significant improvement with BIIB074 over placebo, using a one-sided test at the $5 \%$ level of significance.

The modified intention-to-treat (mITT) population included all patients randomised into the double-blind phase of the study who received at least one dose of double-blind medication (pre-specified population for the primary analysis). The safety population included all patients who received one or more doses of BIIB074. Patients experiencing treatment failure were withdrawn from study treatment, and assessments subsequent to failure were excluded from statistical analyses. All statistical tests were one-sided (assessing improvement with BIIB074 vs placebo) and performed at the 5\% level of significance. No adjustments were made for multiplicity.

The primary analysis compared the number of treatment failures with BIIB074 versus placebo in the mITT population using Fisher's exact test. Secondary efficacy analyses with the primary endpoint included treatment failures by week and time to treatment failure. Time to treatment failure was plotted using Kaplan-Meier curves and treatment groups were compared using the log-rank test. The secondary endpoints relating to paroxysms (number 
TN Phase 2

and severity of paroxysms, and average daily pain score) were analysed using general estimating equation (GEE) linear regression models (compound symmetry covariance structure and log-link function). The change in each endpoint over 4 weeks of treatment was estimated and compared between groups. The PGIC and CGIC scores were analysed using the Wilcoxon rank-sum test. Changes from baseline were summarised, with baseline taken as run-in for the open-label period and Day 21 (end of open-label) for the double-blind period. Pharmacokinetic parameters were estimated using non-linear mixed effect methodology and prior pharmacokinetic knowledge of the compound.

Post-hoc analyses were also conducted for: 1) percentage change from baseline in number of paroxysms of pain at the end of the double-blind phase, 2) change from baseline in paroxysm severity at the end of the double-blind phase, and 3) change from baseline in average daily pain score at the end of the double-blind phase. For each endpoint, the run-in week was taken as baseline and two approaches were used for imputing data: last observation carried forward (LOCF) and baseline observation carried forward (BOCF). Comparisons were made between treatment groups using analysis of covariance. We note that all methods of dealing with missing values have their limitations, ${ }^{23}$ and it is debated whether BOCF and LOCF are good estimates for the missing values; they are also criticized for being single value imputations, and hence underestimate the variability of treatment comparisons. Unfortunately other methods, such as multiple imputation analysis, are likely to have been problematic with the small sample size in this study.

Additionally, the proportion of patients with $\geq 50 \%$ reduction in each of these measures (number of paroxysms, severity of paroxysms, and average daily pain score) was calculated for each treatment group (a 50\% threshold was selected as commonly accepted in the pain literature as reflective of an effective treatment ${ }^{24}$ ). Additional details of statistical analyses are included in the appendix. All analyses were conducted using SAS (version 9.1.3 or later).

The DMC reviewed the data after the first 10 patients had completed the open-label phase. This review did not involve an interim analysis of the primary endpoint and therefore 
TN Phase 2

had no impact on the statistical properties of the study. Details concerning the purpose of the review are included in the appendix. Important changes to the protocol during the study are also detailed in the appendix.

This study is registered on ClinicalTrials.gov (NCT01540630) and EudraCT (2010-02396316).

\section{Role of the funding source}

The funder was involved in study design, data collection, data analysis, data interpretation, and writing of the report. The corresponding author had full access to all the data in the study and had final responsibility for the decision to submit for publication.

\section{Results}

The first patient was enrolled in the study on April 23, 2012, and the last patient completed on February 26, 2014. Overall, 125 patients were screened (primarily to ensure medical fitness), and the diagnostic committee reviewed the trigeminal neuralgia diagnosis for 104 (all those not reviewed were screen failures). The diagnostic committee confirmed the diagnosis in 97 patients; 7 patients from 4 sites were excluded as they did not fulfil the criteria set by the DMC (figure 1). A total of 67 patients entered the open-label phase, all of whom had a confirmed diagnosis prior to entry.

Forty-four (66\%) of 67 patients completed the open-label phase. Of these patients, 30 met the randomisation criteria and were randomised to the double-blind phase, but one was withdrawn prior to dosing because study medication was mislaid at the study site and could not be dispensed. The mITT population therefore included 29 patients who received treatment ( $n=15$ BIIB074, $n=14$ placebo) (figure 1).

The median age of the overall open-label population $(n=67)$ was 60.0 years (range 21-79 years) and $65.7 \%$ were female. Median duration of trigeminal neuralgia was 6.0 years, and median number of previous therapies was two. Characteristics were similar between 
TN Phase 2

treatment groups in the double-blind phase, except for a slightly higher proportion of females in the BIIB074 group (table 1). 98.5\% of patients had received at least one prior medication for trigeminal neuralgia on entering the study; only one patient was drug naïve. Sodium channel blockers were the most commonly used; $74.6 \%$ had used carbamazepine (median daily dose $600 \mathrm{mg}$ ), and $28.4 \%$ oxcarbazepine (median daily dose $900 \mathrm{mg}$ ), during their lifetime. Gabapentin had been used by $31.3 \%$ (median daily dose $1050 \mathrm{mg}$ ) (table 1).

At the time of screening, $50.7 \%$ were receiving carbamazepine, and $22.4 \%$ oxcarbazepine, and had these medications stopped prior to the open-label phase (table 1). Overall, $26.9 \%$ of patients took at least one concomitant medication for trigeminal neuralgia during study treatment. Among patients in the double-blind population, $33.3 \%(n=5$ of 15$)$ in the BIIB074 group and $28.6 \%(n=4$ of 14$)$ in the placebo group reported one or more concomitant medication. In all cases, the most common medication was gabapentin $(19.4 \%$ open-label, 26.7\% BIIB074 double-blind, and 21.4\% placebo double-blind) (table 1).

During the double-blind phase, fewer patients receiving BIIB074 experienced treatment failure ( $n=5$ of $15 ; 33.3 \%)$ than with placebo $(n=9$ of $14 ; 64.3 \%)$; however, the difference between groups was not statistically significant (one-sided $p=0.0974$ primary endpoint). A breakdown of patients meeting individual treatment failure criteria during the double-blind phase is reported in the appendix (table S2). The majority of treatment failures occurred in the first 2 weeks of double-blind treatment (data not shown).

The median time to treatment failure during the double-blind phase was significantly longer with BIIB074 than placebo (one-sided $p=0.0306$; figure 2). For BIIB074, less than $50 \%$ experienced treatment failure, and thus a median time to failure was not reached (NR; $95 \% \mathrm{Cl} 7.0, \mathrm{NR})$. In the placebo group, the median time to treatment failure was 14.0 days $(95 \% \mathrm{Cl} 1.0, \mathrm{NR})$.

During run-in, patients in the mITT population experienced a median of 7.9 (range 2.4 to 51.0 ) paroxysms per day, mean severity of paroxysms of 5.7 (SD 1.47), and mean average daily pain score of 5.7 (SD 1.61). At the end of the open-label phase, these measures were reduced to a median of 3.9 (range 0-39.6) paroxysms per day, mean 
TN Phase 2

severity of paroxysms of 2.8 (SD 2.19) points, and mean average daily pain score of 2.6 (SD 2.17). A GEE model comparing reductions during double-blind treatment with BIIB074 relative to placebo estimated a $45 \%$ placebo-adjusted reduction in the number of paroxysms (95\% Cl $-2 \%, 70 \%$; one-sided $\mathrm{p}=0.028)$, a $26 \%$ placebo-adjusted reduction in severity of paroxysms (95\% Cl -13\%, 51\%; one-sided $\mathrm{p}=0.0846)$, and a $50 \%$ placebo-adjusted reduction in average daily pain score (95\% $\mathrm{Cl} 23 \%, 67 \%$; one-sided $\mathrm{p}=0.0009)$.

In a post-hoc analysis (BOCF), the number of paroxysms was reduced by a mean of $53 \%$ from run-in to the end of double-blind treatment in the BlIB074 group compared with $21 \%$ in the placebo group (placebo-adjusted change $-32 \%, 95 \% \mathrm{Cl}-64 \%, 1 \%$ ). Severity of paroxysms was reduced by a mean of 2.49 points with BIIB074 compared with 1.13 with placebo (placebo-adjusted change $-1.35,95 \% \mathrm{Cl}-3.06,0.35)$. The mean reduction in average daily pain score was 3.05 in the BIIB074 group and 0.74 in the placebo group (placebo-adjusted change $-2.31,95 \% \mathrm{Cl}-3.78,-0.83$ ). Figure 3 plots the mean percentage change in these pain measures from run-in across the course of the study (BOCF). We report BOCF data as we believe the most reasonable assumption is that pain levels return to baseline on cessation of treatment (BOCF was also recommended for randomised withdrawal trials in a recent systematic review ${ }^{25}$ ), but analysis by LOCF showed similar results (data not shown).

An additional post-hoc analysis evaluated the percentage of patients experiencing a $\geq 50 \%$ reduction in number of paroxysms, severity of paroxysms, or average daily pain score from run-in to the end of the double-blind phase. The number of paroxysms was reduced by $\geq 50 \%$ for $60.0 \%$ ( $n=9$ of 15 ) of the BIIB074 group compared with $21.4 \%$ ( $n=3$ of 14 ) of the placebo group, and severity of paroxysms was reduced by $\geq 50 \%$ for $46.7 \%$ ( $n=7$ of 15 ) receiving BIIB074 compared with $14.3 \%$ ( $n=2$ of 14 ) receiving placebo. $A \geq 50 \%$ reduction in average daily pain score was experienced by $60.0 \%$ ( $n=9$ of 15$)$ in the BIIB074 group compared with $14.3 \%(n=2$ of 14$)$ in the placebo group.

All patients in the mITT population had improvement (minimally improved, much improved or very much improved) according to CGIC during the open-label phase ( $n=29$ of 
TN Phase 2

$29 ; 100.0 \%$ ), and all but one had improvement on the PGIC ( $n=28$ of $29 ; 96.6 \%)$. At the end of double-blind treatment, CGIC indicated further improvement relative to Day 21 for $80.0 \%$ ( $n=12$ of 15$)$ of BllB074 patients versus $35.7 \%(n=5$ of 14$)$ of placebo patients. The proportion of patients reporting improvement in PGIC was $73.3 \%(n=11$ of 15$)$ in the BIIB074 group versus $50.0 \%$ ( $n=7$ of 14$)$ in the placebo group. One-sided $p$-values from Wilcoxon exact tests, using data over the whole scale, were 0.0051 and 0.0265 for CGIC and PGIC, respectively, for BIIB074 vs placebo.

During the open-label phase, 37 of 67 (55.2\%) patients reported a total of 139 AEs (68 considered treatment-related [includes unknown, probable, possible, or definite causality]). During the double-blind phase, 4 of 15 (26.7\%) patients in the BlIB074 group reported 13 treatment-emergent AEs (new AEs or worsening of AEs from the open-label phase) (seven treatment-related), and 5 of 14 (35.7\%) in the placebo group reported 17 treatment-emergent AEs (5 treatment-related). Only 8 of $152(5.3 \%)$ AE events while receiving BIIB074 were considered severe, experienced by 5 patients receiving BIIB074 during open label treatment. For 3 of these patients, the AEs (trigeminal neuralgia pain [also reported as a serious adverse event (SAE)], worsening of trigeminal neuralgia, and headache [verbatim terms]) were considered by the investigator to be unrelated to BIIB074. Severe AEs of headache, extremely decreased skin turgor, and extremely dry mouth reported for one patient during open-label treatment with BIIB074 were all considered probably related to study treatment. One patient had 2 severe events of dizziness that were also considered probably related to treatment with BIIB074. One patient randomised to placebo had a severe AE of intestinal adhesions with obstruction (reported as an SAE) shortly after discontinuing double-blind treatment due to lack of efficacy. The event was considered unrelated to study treatment.

In addition to the two SAEs described above (trigeminal neuralgia pain and intestinal adhesions), an SAE of food poisoning with Escherichia coli was reported during open-label treatment, which was not considered to be treatment-related. Five patients had treatment withdrawn or interrupted due to AEs, including two of the patients with SAEs (trigeminal 
TN Phase 2

neuralgia pain and Escherichia coli - the patients were subsequently withdrawn from the study due to lack of efficacy and withdrawal of consent), and the patient with severe AEs of decreased skin turgor and dry mouth, during open-label treatment. Two additional patients with AEs of hypertension (probably related) and dyspnoea (unrelated) during the open-label phase were withdrawn due to these AEs. No patients discontinued due to AEs during the double-blind phase. The most common AEs reported during the open-label and double-blind phases ( $\geq 3.0 \%$ overall incidence) are listed in table 2 , with very few relating to impaired cognitive functioning. There were no clinically significant AEs in relation to laboratory safety tests (including urea and electrolytes and liver function tests), vital signs, or ECG.

BIIB074 steady state plasma concentrations and exposures were as expected from the prior studies in healthy volunteers. Estimated individual $\mathrm{C}_{\text {min-ss }}$ and $\mathrm{C}_{\text {max-ss }}$ values suggested that plasma concentrations with BIIB074 $150 \mathrm{mg}$ tid remained between approximately 0.5 and $2.7 \mu \mathrm{g} / \mathrm{mL}$, without obvious differences between randomisation groups or periods. The estimated steady state exposures corresponded to an estimated mean $\mathrm{C}_{\max }$ of $2 \mu \mathrm{g} / \mathrm{mL}$ and mean $\mathrm{AUC}_{0-24}$ of $36 \mu \mathrm{g} \cdot \mathrm{h} / \mathrm{ml}$.

During assessment of masking at the end of double-blind treatment, $66.7 \%$ of patients ( $n=10$ of 15$)$ and clinicians for $73.3 \%$ of patients $(n=11$ of 15$)$ in the BIIB074 group guessed their treatment allocation correctly. In the placebo group, patients and clinicians each guessed correctly for $71.4 \%$ of patients $(n=10$ of 14 ). It should be noted that these assessments are probably influenced by effects of the drug on pain and AEs.

\section{Discussion}

Fewer patients treated with the Nav1.7 blocker BIIB074 had treatment failures, the primary endpoint of the study, than those given placebo in the double-blind phase of the study, but the difference was not statistically significant. Statistically significant treatment differences were observed versus placebo in important secondary endpoints during the double-blind phase, including time to treatment failure, number of paroxysms, average daily pain score, 
TN Phase 2

PGIC, and CGIC. While firm conclusions on efficacy and safety cannot be drawn from this study, the results provide a basis for continued study.

BIIB074 at a dose of $150 \mathrm{mg}$ tid was well-tolerated in this study, with few severe AEs or withdrawals due to AEs. During the double-blind phase, the incidence of AEs was similar with both BIIB074 and placebo. The most common AEs in the open-label phase were headache and dizziness, occurring in $19.4 \%$ and $9.0 \%$ of patients, respectively. This incidence might reasonably be expected in any population. In the double-blind phase, no AE (new or worsening from open-label) occurred in more than one patient. While direct comparisons with $\mathrm{AE}$ percentages for other drugs cannot be made, overall the tolerability profile appears promising relative to carbamazepine, oxcarbazepine, and other anticonvulsants used in trigeminal neuralgia, which exhibit high rates of cognitive and other central nervous system (CNS) events..$^{3-5,7,26-28}$ In contrast, BIIB074 was associated with low rates of these AEs, and in particular cognitive impairment and drowsiness. Further evaluation of the AE profile of BIIB074 relative to carbamazepine and oxcarbazepine will be important. Given the good tolerability profile of BIIB074, it may also be possible to increase the dose to determine if efficacy could be improved. Indeed, higher doses have been evaluated in Phase 1 studies and a Phase 2 trial in patients with painful lumbosacral radiculopathy, and the $A E$ profile was broadly similar to the current study (unpublished; ClinicalTrials.gov Identifiers: NCT00908154, NCT00955396 (Phase 1); NCT01561027 [Phase 2]).

Pain in trigeminal neuralgia is thought to arise from abnormal action potential discharges from trigeminal ganglion neurons. ${ }^{18,19}$ Aligning with the promising clinical results, in vitro electrophysiological studies (shown in the online appendix, page 3 and figure S3) show an inhibitory effect of BIIB074 on the excitability of trigeminal ganglion neurons, including a reduction in high-frequency firing. Of particular note, the maximum serum concentration of BIIB074 observed in the present trial and another Phase 2 study in painful lumbosacral radiculopathy (unpublished; ClinicalTrials.gov Identifier: NCT01561027) was in the same order of magnitude as the lower concentration tested in electrophysiology studies 
TN Phase 2

$(1 \mu \mathrm{mol} / \mathrm{L})$, indicating the clinical relevance of this dose. We cannot exclude a contribution of blockade of sodium channels within the CNS to the actions of BIIB074. Nonetheless, our observations demonstrate a direct inhibitory action of BIIB074 on trigeminal ganglion neurons. Together with earlier work demonstrating the presence of Nav1.7 in trigeminal ganglion neurons, ${ }^{10,11}$ selectivity of BIIB074 for Nav1.7, and a frequency-dependent effect, ${ }^{15-}$

${ }^{17}$ these data further suggest the potential for BIIB074 in trigeminal neuralgia and suggest a probable mechanism of action at the site of ectopic impulse activity in trigeminal neuralgia. Importantly, a selective Nav1.7 inhibitor also has potential from a mechanistic perspective to be effective in other forms of neuropathic pain, such as erythromelalgia, which has been shown to be reduced by pharmacological inhibition of Nav1.7. ${ }^{29}$ BIIB074 is currently being investigated in patients with erythromelalgia and has also previously shown a statistically significant reduction in pain relative to placebo in a Phase 2 trial in patients with painful lumbosacral radiculopathy (data on file).

This study utilised a randomised withdrawal design. The critical aspect of this design in relation to trigeminal neuralgia is that it exposes patients with severe pain to placebo for the minimum possible time..$^{20,30}$ This also avoids problems with the alternative of using an active control such as carbamazepine (e.g. potential for drug-drug interactions, requirement for long washout period). While the randomised withdrawal design has been employed previously in pain studies, ${ }^{25}$ to our knowledge this is the first time that it has been used in a trigeminal neuralgia population. In a recent systematic review of randomised withdrawal trials, Moore et $\mathrm{al}^{25}$ suggested that this methodology is acceptable for trials to address efficacy of drugs in chronic non-cancer pain. In our judgement, this trial further supports the feasibility of the randomised withdrawal design to evaluate a novel drug in trigeminal neuralgia.

Other key considerations in the design of this study included ensuring diagnostic accuracy and homogeneity of the study population. In this regard, we included a diagnostic sub-committee of the DMC to ensure that ICHD criteria for classical trigeminal neuralgia were met by all patients entering the study. Patients with pain attributable to other disorders 
TN Phase 2

were excluded. Patients were also required to experience at least three paroxysms of pain per day, pain intensity $\geq 4$ (PI-NRS) on at least 4 days during run-in, in order to be eligible for inclusion. Further care was taken to select the most appropriate outcome measures for this population, with the inclusion of both pain measures and assessments of overall function and quality of life (PGIC, CGIC), which can be severely impacted in trigeminal neuralgia.

Of course, while carefully designed, this trial remains a small phase 2 study and as such firm conclusions on efficacy and safety cannot be drawn. The randomised withdrawal design has several inherent limitations. For example, there is a risk of bias on transition from the open-label to double-blind phase because patients have prior experience of active treatment, and those switching to placebo may be aware of the change due to loss of effects of the drug on pain or side effects. Indeed, $67-73 \%$ of patients and clinicians in both treatment groups guessed their treatment assignment correctly. There is also the possibility of carry-over effects from active treatment in patients switched to placebo, which may make it more difficult to achieve separation between groups in the double-blind phase. The enrichment of the study population through the selection of responders in the open-label phase also inherently reduces generalizability of the data. A further limitation from a functional perspective was that per investigator report patients found it difficult to record in the paper diary the number of paroxysms per day (particularly if these were very frequent); an electronic diary would be more reliable and may be considered for future studies.

An additional point of note was the substantial drop in patient numbers from screening to the end of the open-label phase of this study. Reasons for this probably included regulatory requirements for ECG parameters (exclusion of patients with prolonged QT interval), leading to exclusions given the older age of the population (up to 80 years). Some patients with severe pain may also have elected to undergo surgery in place of entering the trial or have been reluctant to stop their current anticonvulsants for fear of pain increase and the need to return to their old medications after completion of the trial. A separate paper is in preparation discussing the challenges in recruitment in this study and trigeminal neuralgia trials more broadly. Further, a relatively high proportion of patients who 
TN Phase 2

were enrolled did not complete the open-label phase (23 overall, 18 withdrawing due to 'lack of efficacy'. Many of these patients withdrew in the first few days, and while precise reasons were not documented, it is possible that their reluctance to proceed was related to anxiety following discontinuation of their prior medications. This will be partially addressed in a planned Phase 3 trial by allowing concomitant use of ongoing medications alongside BIIB074 for the first 2 weeks.

Most drugs currently in use to treat this disorder were originally trialled as anticonvulsant therapy; to our knowledge BIIB074 is unique in being developed for trigeminal neuralgia as the primary indication. Despite the limitations of our study, we believe that the results provide a basis for the continued investigation of BIIB074 in trigeminal neuralgia. The results from this Phase 2a study have been used to develop and refine the design for a forthcoming Phase 3 trial to assess BIIB074 in trigeminal neuralgia, utilizing the randomized withdrawal design, and with an increased sample size and longer duration of dosing. 
TN Phase 2

\section{Contributors}

JZ, JP, VM, MO, DE, GC, LB, KG and ST contributed to the design, and JZ, JP, VM, GG, MO, DE, GC, LB, KG and ST to the conduct of the Phase 2a study. VM, ME, DD and SGW designed and conducted the preclinical electrophysiological studies reported. GL was responsible for statistical review of the data analyses. All authors contributed to the interpretation of the study results and to the review and critical revision of the article. All authors approved the final version of the article for submission.

\section{Declaration of interests}

The preclinical work described was supported by a grant from Convergence Pharmaceuticals to the laboratory of Stephen Waxman and Mark Estacion at Yale University. Drs Waxman and Estacion have no other conflicts of interest to declare. Joanna Zakrzewska was supported by the National Institute for Health Research University College London Hospitals Biomedical Research Centre and also received a consultancy fee from Convergence Pharmaceuticals. Mark Obermann has received scientific support and/or honoraria from Convergence Pharmaceuticals, Biogen Idec, Novartis, Sanofi-Aventis, Genzyme, Pfizer, Teva, Johnson \& Johnson, and Heel. He received research grants from Heel, Allergan, Electrocore, and the German Ministry for Education and Research (BMBF). Dominik Ettlin's institution received payment for conducting the study and Dr Ettlin received a consultancy fee from Convergence Pharmaceuticals. Giorgio Cruccu has received financial support and honoraria from Astellas, Biogen-Convergence, and Sigma Tau. Lars Bendtsen's institution received payment for conducting the study, however, Dr Bendtsen had no personal financial interest and has no other conflicts of interests to declare with regard to this study. Joanne Palmer, Valerie Morisset, Gerard Giblin, Dominique Derjean, and Simon Tate are employees and were shareholders of Convergence Pharmaceuticals. Kevin Gunn was an employee of Convergence Pharmaceuticals at the time this study was conducted and was a shareholder in Convergence Pharmaceuticals. Gary Layton is an employee of 
TN Phase 2

ParamStat Ltd, paid consultants to Convergence Pharmaceuticals in connection with this study and other work.

\section{Acknowledgements}

The Phase 2a study was funded by Convergence Pharmaceuticals Ltd., a Biogen company. Biogen provided funding for medical writing support in the development of this manuscript. Elizabeth Harvey, PhD (Excel Scientific Solutions, Hammersmith, London, UK), wrote the first draft of the manuscript based on input from authors. Biogen reviewed and provided feedback on the paper. The authors had full editorial control of the paper, and provided their final approval of all content. The authors thank all of the study investigators and the patients who participated in this study. The authors also thank Giulia Di Stefano (Sapienza University) and Stine Maarbjerg (University of Copenhagen) for their help in conducting the study. Thanks also go to the reviewers for their helpful comments. 
TN Phase 2

\section{References}

1. International Headache Society. The International Classification of Headache Disorders, 3rd edition (beta version). Cephalalgia. 2013; 33(9): 629-808.

2. Cruccu G, Gronseth G, Alksne J, Argoff C, Brainin M, Burchiel K, et al. AAN-EFNS guidelines on trigeminal neuralgia management. Eur J Neurol. 2008; 15(10): 1013-28.

3. Wiffen PJ, Derry S, Moore RA, Kalso EA. Carbamazepine for chronic neuropathic pain and fibromyalgia in adults. Cochrane Database Syst Rev. 2014; 4: CD005451.

4. Di Stefano G, La Cesa S, Truini A, Cruccu G. Natural history and outcome of 200 outpatients with classical trigeminal neuralgia treated with carbamazepine or oxcarbazepine in a tertiary centre for neuropathic pain. J Headache Pain. 2014; 15: 34.

5. Besi E, Boniface DR, Cregg R, Zakrzewska JM. Comparison of tolerability and adverse symptoms in oxcarbazepine and carbamazepine in the treatment of trigeminal neuralgia and neuralgiform headaches using the Liverpool Adverse Events Profile (AEP). J Headache Pain. 2015; 16: 563.

6. Zhang J, Yang M, Zhou M, He L, Chen N, Zakrzewska JM. Non-antiepileptic drugs for trigeminal neuralgia. Cochrane Database Syst Rev. 2013; 12: CD004029.

7. Wiffen PJ, Derry S, Moore RA, Aldington D, Cole P, Rice AS, et al. Antiepileptic drugs for neuropathic pain and fibromyalgia - an overview of Cochrane reviews. Cochrane Database Syst Rev. 2013; 11: CD010567.

8. Zakrzewska JM, Linskey ME. Trigeminal neuralgia. BMJ Clin Evid. 2014; 2014.

9. Mittal SO, Safarpour D, Jabbari B. Botulinum Toxin Treatment of Neuropathic Pain. Semin Neurol. 2016; 36(1): 73-83.

10. Dib-Hajj SD, Yang Y, Black JA, Waxman SG. The Na(V)1.7 sodium channel: from molecule to man. Nat Rev Neurosci. 2013; 14(1): 49-62.

11. Dib-Hajj SD, Cummins TR, Black JA, Waxman SG. Sodium channels in normal and pathological pain. Annu Rev Neurosci. 2010; 33: 325-47.

12. Bagal SK, Chapman ML, Marron BE, Prime R, Storer RI, Swain NA. Recent progress in sodium channel modulators for pain. Bioorg Med Chem Lett. 2014; 24(16): 3690-9. 
TN Phase 2

13. Cox JJ, Reimann F, Nicholas AK, Thornton G, Roberts E, Springell K, et al. An SCN9A channelopathy causes congenital inability to experience pain. Nature. 2006; 444(7121): 894-8.

14. Jo S, Bean BP. Sidedness of carbamazepine accessibility to voltage-gated sodium channels. Mol Pharmacol. 2014; 85(2): 381-7.

15. Tate S, Davis JB, Owen D, Derjean D, Rugiero F, Giblin GM, et al. CNV1014802 a novel potent state-dependent sodium channel blocker with broad preclinical antihyperalgesic efficacy. Program No. 275.06/LL2. 2011 Neuroscience Meeting Planner. Washington, DC: Society for Neuroscience, 2011. Online. 2011.

16. Morisset V, Davis JB, Tate SN. Mechanism of Action of Anticonvulsants as Analgesic Drugs. Textbook of Pain, 6th Edition (Elsevier) Chapter 35, p491-499. 2013.

17. Rugiero F, Derjean D, Morisset V, Owen DE, Giblin GMP, Davis JB, et al. CNV1014802, a novel, potent Nav1.7 selective state-dependent sodium channel blocker with broad preclinical antihyperalgesic efficacy. Presented at 14th World Congress on Pain (IASP), Milan, Italy. 2012.

18. Burchiel KJ, Baumann TK. Pathophysiology of trigeminal neuralgia: new evidence from a trigeminal ganglion intraoperative microneurographic recording. Case report. J Neurosurg. 2004; 101(5): 872-3.

19. Rappaport ZH, Devor M. Trigeminal neuralgia: the role of self-sustaining discharge in the trigeminal ganglion. Pain. 1994; 56(2): 127-38.

20. Zakrzewska JM, Palmer J, Ettlin DA, Obermann M, Giblin GM, Morisset V, et al. Novel design for a phase lla placebo-controlled, double-blind randomized withdrawal study to evaluate the safety and efficacy of CNV1014802 in patients with trigeminal neuralgia. Trials. 2013; 14: 402.

21. International Headache Society. The International Classification of Headache Disorders: 2nd edition. Cephalalgia. 2004; 24 Suppl 1: 9-160. 
TN Phase 2

22. Lee JY, Chen HI, Urban C, Hojat A, Church E, Xie SX, et al. Development of and psychometric testing for the Brief Pain Inventory-Facial in patients with facial pain syndromes. J Neurosurg. 2010; 113(3): 516-23.

23. Sterne JA, White IR, Carlin JB, Spratt M, Royston P, Kenward MG, et al. Multiple imputation for missing data in epidemiological and clinical research: potential and pitfalls. BMJ. 2009; 338: b2393.

24. Dworkin RH, Turk DC, Wyrwich KW, Beaton D, Cleeland CS, Farrar JT, et al. Interpreting the clinical importance of treatment outcomes in chronic pain clinical trials: IMMPACT recommendations. J Pain. 2008; 9(2): 105-21.

25. Moore RA, Wiffen PJ, Eccleston C, Derry S, Baron R, Bell RF, et al. Systematic review of enriched enrolment, randomised withdrawal trial designs in chronic pain: a new framework for design and reporting. Pain. 2015; 156(8): 1382-95.

26. Meador KJ. Cognitive and memory effects of the new antiepileptic drugs. Epilepsy Res. 2006; 68(1): 63-7.

27. Meador KJ, Gevins A, Loring DW, McEvoy LK, Ray PG, Smith ME, et al. Neuropsychological and neurophysiologic effects of carbamazepine and levetiracetam. Neurology. 2007; 69(22): 2076-84.

28. Motamedi GK, Meador KJ. Antiepileptic drugs and memory. Epilepsy Behav. 2004; 5(4): 435-9.

29. Cao L, McDonnell A, Nitzsche A, Alexandrou A, Saintot PP, Loucif AJ, et al. Pharmacological reversal of a pain phenotype in iPSC-derived sensory neurons and patients with inherited erythromelalgia. Sci Transl Med. 2016; 8(335): 335ra56.

30. FDA Guidance for Industry. E 10 Choice of Control Group and Related Issues in Clinical Trials.

http://wwwfdagov/downloads/drugs/guidancecomplianceregulatoryinformation/guidances/uc m073139pdf. 
Table 1. Baseline demographics and trigeminal neuralgia history and medications

\begin{tabular}{|c|c|c|c|c|}
\hline & & \multirow{2}{*}{\begin{tabular}{|l|} 
Open-label phase \\
BllB074 \\
$(\mathrm{N}=67)$
\end{tabular}} & \multicolumn{2}{|c|}{ Double-blind phase } \\
\hline & & & $\begin{array}{l}\text { BllB074 } \\
(\mathrm{N}=15)\end{array}$ & $\begin{array}{l}\text { Placebo } \\
(\mathrm{N}=14)\end{array}$ \\
\hline Age, years & Median (range) & $60.0(21-79)$ & $52.0(26-72)$ & $56.5(21-74)$ \\
\hline \multirow[t]{2}{*}{ Sex, n (\%) } & Male & $23(34.3)$ & $4(26.7)$ & $6(42.9)$ \\
\hline & Female & $44(65.7)$ & $11(73.3)$ & $8(57.1)$ \\
\hline TN duration, years & Median (range) & $6.0(0-35)$ & $4.0(1-17)$ & $7.0(1-11)$ \\
\hline Previous TN therapies, $\mathrm{n}$ & Median (range) & $2.0(1-25)$ & $1.0(1-6)$ & $2.0(1-5)$ \\
\hline \multirow[t]{6}{*}{ Anatomical site of TN pain, $n(\%)$} & $1^{\text {st }}$ branch & $1(1.5)$ & 0 & $1(7.1)$ \\
\hline & $2^{\text {nd }}$ branch & $17(25.4)$ & $5(33.3)$ & $4(28.6)$ \\
\hline & $3^{\text {rd }}$ branch & $17(25.4)$ & $6(40.0)$ & $1(7.1)$ \\
\hline & $1^{\text {st }}$ and $2^{\text {nd }}$ branch & $5(7.5)$ & $1(6.7)$ & $2(14.3)$ \\
\hline & $1^{\text {st }}, 2^{\text {nd }}$ and $3^{\text {rd }}$ branch & $7(10.4)$ & $1(6.7)$ & $1(7.1)$ \\
\hline & $2^{\text {nd }}$ and $3^{\text {rd }}$ branch & $20(29.9)$ & $2(13.3)$ & $5(35.7)$ \\
\hline Use of TN medications during & Any, $\mathrm{n}(\%)$ & $66(98.5)$ & $14(93.3)$ & $14(100)$ \\
\hline
\end{tabular}




\begin{tabular}{|c|c|c|c|c|}
\hline \multirow[t]{6}{*}{ lifetime } & Carbamazepine, n (\%) & $50(74.6)$ & $10(66.7)$ & $11(78.6)$ \\
\hline & $\begin{array}{l}\text { Median daily dose } \\
\text { (range), mg }\end{array}$ & $\begin{array}{l}N=49^{\#} \\
600(200-1600)\end{array}$ & $\begin{array}{l}N=10 \\
600(200-1600)\end{array}$ & $\begin{array}{l}\mathrm{N}=10^{\#} \\
600(300-800)\end{array}$ \\
\hline & Oxcarbazepine, n (\%) & $19(28.4)$ & $5(33.3)$ & $4(28.6)$ \\
\hline & $\begin{array}{l}\text { Median daily dose } \\
\text { (range), mg }\end{array}$ & $900(300-1800)$ & $1200(300-1800)$ & $1200(450-1500)$ \\
\hline & Gabapentin, n (\%) & $21(31.3)$ & $6(40.0)$ & $4(28.6)$ \\
\hline & $\begin{array}{l}\text { Median daily dose } \\
\text { (range), mg }\end{array}$ & $\begin{array}{l}\mathrm{N}=20^{\#} \\
1050(300-3600)\end{array}$ & $\begin{array}{l}\mathrm{N}=6 \\
700(300-3200)\end{array}$ & $\begin{array}{l}\mathrm{N}=4 \\
900(600-3600)\end{array}$ \\
\hline \multirow{3}{*}{$\begin{array}{l}\text { TN medications at screening, } \\
\text { stopped prior to open-label, n (\%) }\end{array}$} & Any & $52(77.6)$ & $9(60.0)$ & $10(71.4)$ \\
\hline & Carbamazepine & $34(50.7)$ & $6(40.0)$ & $6(42.9)$ \\
\hline & Oxcarbazepine & $15(22.4)$ & $3(20.0)$ & $4(28.6)$ \\
\hline \multirow{2}{*}{$\begin{array}{l}\text { Concomitant TN medications } \\
\text { during trial, } \mathrm{n}(\%)\end{array}$} & Any & $18(26.9)$ & $5(33.3)$ & $4(28.6)$ \\
\hline & Gabapentin & $13(19.4)$ & $4(26.7)$ & $3(21.4)$ \\
\hline
\end{tabular}

$\mathrm{TN}=$ trigeminal neuralgia. ${ }^{\#}$ Dose information was missing for some prior medications. 
TN Phase 2

Table 2. Adverse events (preferred terms) with $\geq 3.0 \%$ overall BIIB 074 incidence

\begin{tabular}{|c|c|c|c|c|}
\hline & \multirow{2}{*}{$\begin{array}{l}\text { Open-label } \\
\text { phase* }^{*} \\
\text { BllB074 } \\
(\mathrm{N}=67)\end{array}$} & \multicolumn{2}{|c|}{ Double-blind phase } & \multirow{2}{*}{$\begin{array}{l}\text { Overall (open- } \\
\text { label and } \\
\text { double-blind) } \\
\text { BllB074 ( } N=67)\end{array}$} \\
\hline & & $\begin{array}{l}\text { BllB074 } \\
(\mathrm{N}=15)\end{array}$ & $\begin{array}{l}\text { Placebo } \\
(\mathrm{N}=14)\end{array}$ & \\
\hline Headache & $13(19.4)$ & $1(6.7)$ & $1(7.1)$ & $13(19.4)$ \\
\hline Dizziness & $6(9.0)$ & 0 & $1(7.1)$ & $6(9.0)$ \\
\hline Dyspepsia & $4(6.0)$ & 0 & 0 & $4(6.0)$ \\
\hline Diarrhoea & $4(6.0)$ & 0 & $1(7.1)$ & $4(6.0)$ \\
\hline $\begin{array}{l}\text { Abdominal pain, } \\
\text { upper }\end{array}$ & $4(6.0)$ & 0 & 0 & $4(6.0)$ \\
\hline Fatigue & $4(6.0)$ & 0 & 0 & $4(6.0)$ \\
\hline Pyrexia & $3(4.5)$ & $1(6.7)$ & 0 & $3(4.5)$ \\
\hline Constipation & $3(4.5)$ & 0 & 0 & $3(4.5)$ \\
\hline $\begin{array}{l}\text { Disturbance in } \\
\text { attention }\end{array}$ & $3(4.5)$ & 0 & 0 & $3(4.5)$ \\
\hline Vomiting & $3(4.5)$ & 0 & $1(7.1)$ & $3(4.5)$ \\
\hline Memory impairment & $2(3.0)$ & 0 & 0 & $2(3.0)$ \\
\hline Nasopharyngitis & $1(1.5)$ & $1(6.7)$ & 0 & $2(3.0)$ \\
\hline Sleep disorder & $1(1.5)$ & $1(6.7)$ & 0 & $2(3.0)$ \\
\hline Somnolence & $2(3.0)$ & 0 & 0 & $2(3.0)$ \\
\hline Tremor & $1(1.5)$ & $1(6.7)$ & 0 & $2(3.0)$ \\
\hline Vision blurred & $2(3.0)$ & 0 & 0 & $2(3.0)$ \\
\hline
\end{tabular}

*Trigeminal neuralgia/worsening of trigeminal neuralgia also accounted for two AEs reported during open-label treatment. $\mathrm{AE}=$ adverse event. 
TN Phase 2

\section{Figure Legends}

Figure 1. Trial profile. *One of 13 was recorded as a protocol deviation as double-blind medication was dispensed without the patient being randomised. ${ }^{\dagger} T$ wo completers in the placebo group met the criteria for treatment failure but were not withdrawn from the study in error. $\mathrm{DMC}=$ data monitoring committee; tid=3 times daily.

Figure 2. Kaplan-Meier graph of time to treatment failure during double-blind phase (mITT population, $\mathrm{N}=29$ ). Log-rank test; one-sided $\mathrm{p}=0.0306$. $\mathrm{mlTT}=$ modified intent-totreat.

Figure 3. Mean (SE) percentage change from run-in in (A) number of paroxysms, (B) severity of paroxysms, and (C) average daily pain score during open-label and doubleblind treatment (BOCF; mITT population, $\mathrm{N}=29$; post-hoc analysis). Although all patients received BIIB074 during the open-label period, data are reported separately for patients entering the BIIB074 and placebo groups during double blind treatment (mITT population) in order to follow treatment response for these patients over the full study period. $\mathrm{BOCF}=$ baseline observation carried forward; $\mathrm{mITT}=$ modified intent-to-treat;

$\mathrm{SE}=$ standard error. 


\section{TN Phase 2}

\section{Figure 1. Trial profile}

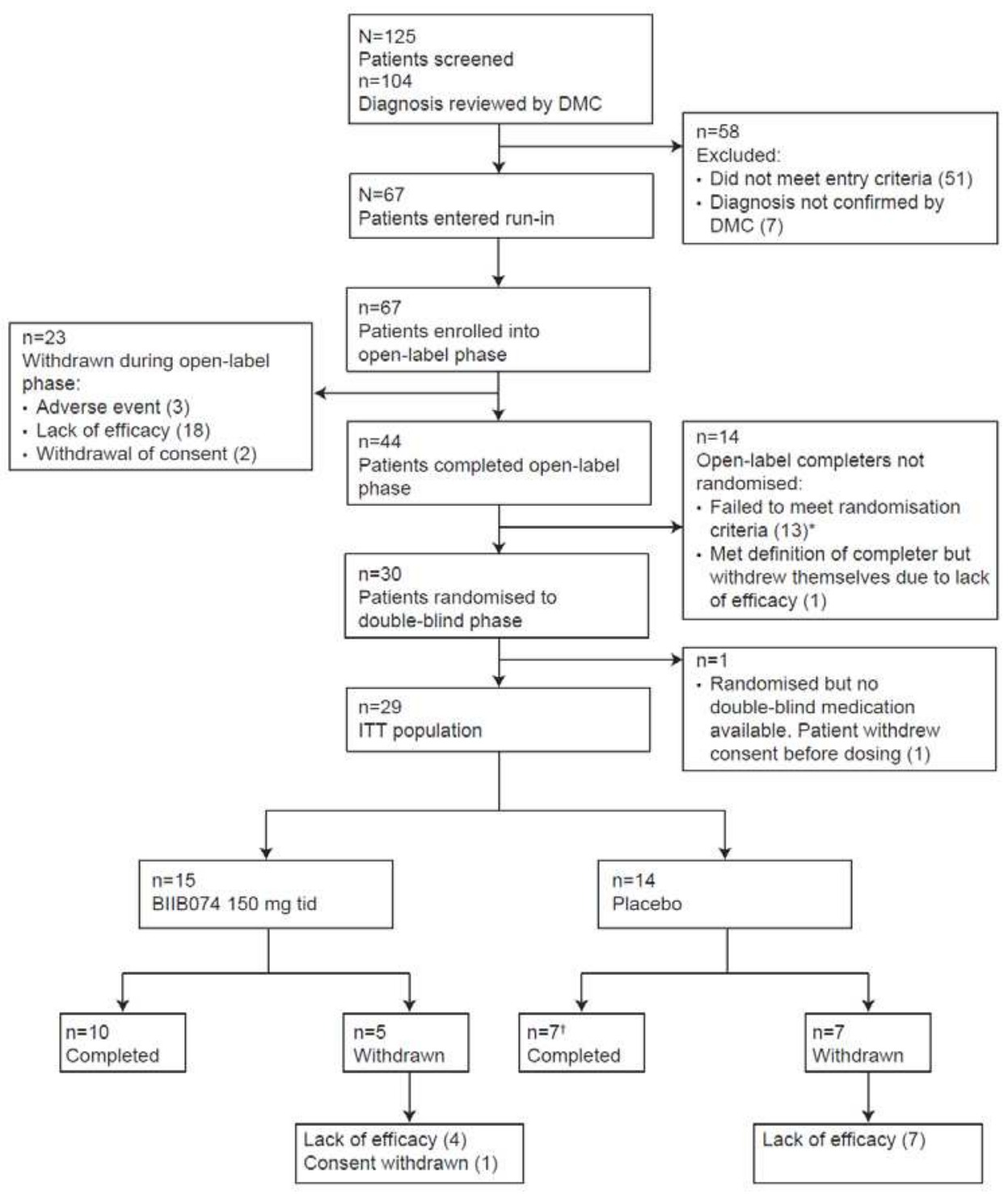


TN Phase 2

Figure 2. Kaplan-Meier plot of time to treatment failure during double-blind phase (mITT population, $\mathrm{N}=29$ )

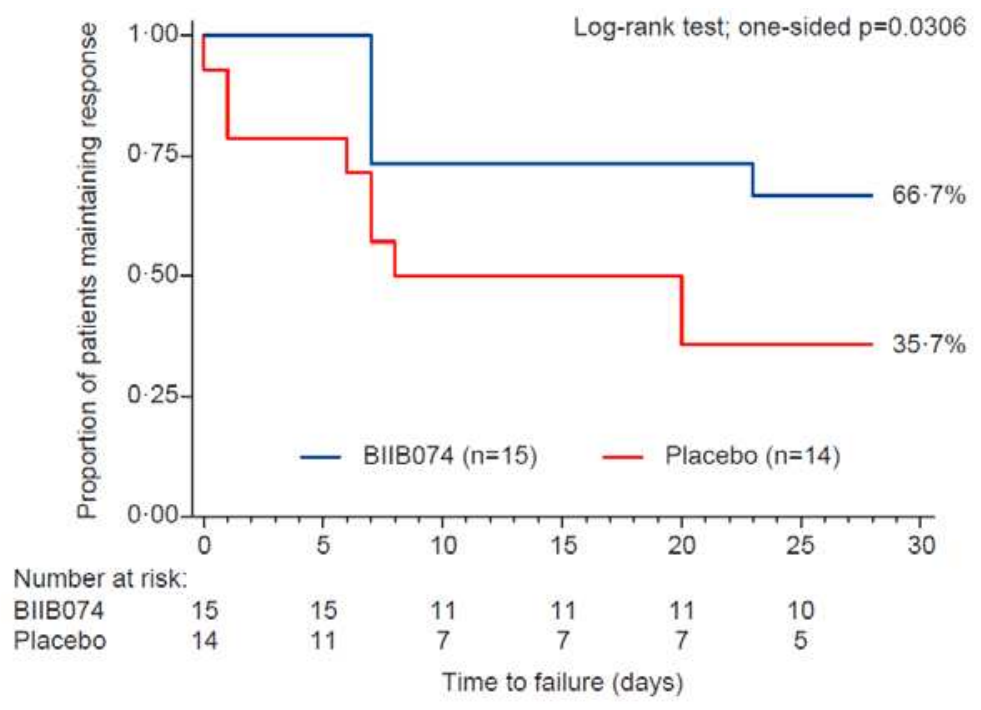


TN Phase 2

Figure 3. Mean (SE) percentage change from run-in in (A) number of paroxysms,

(B) severity of paroxysms, and (C) average daily pain score during open-label and double-blind treatment (BOCF; mITT population, $\mathrm{N}=29$; post-hoc analysis).

A

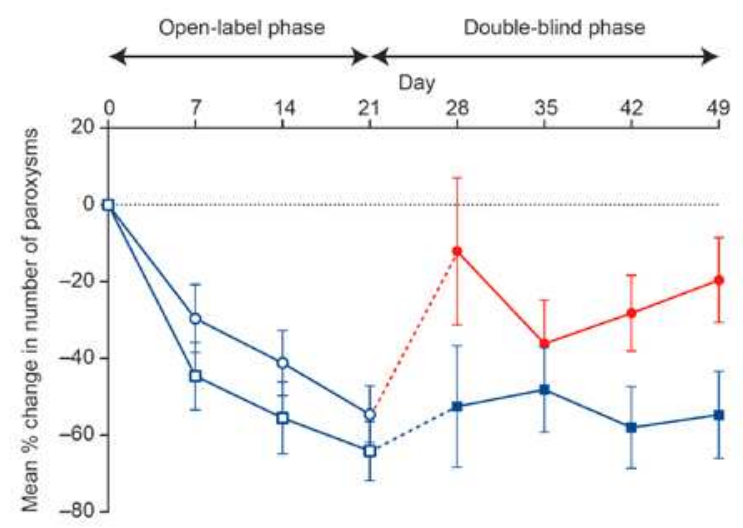

B

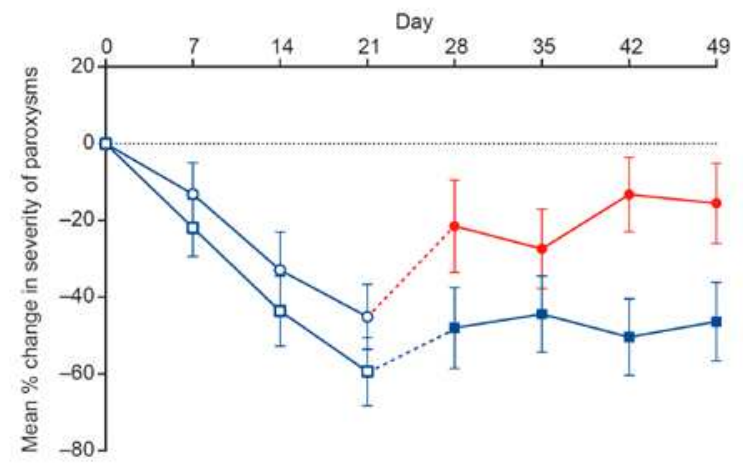

c

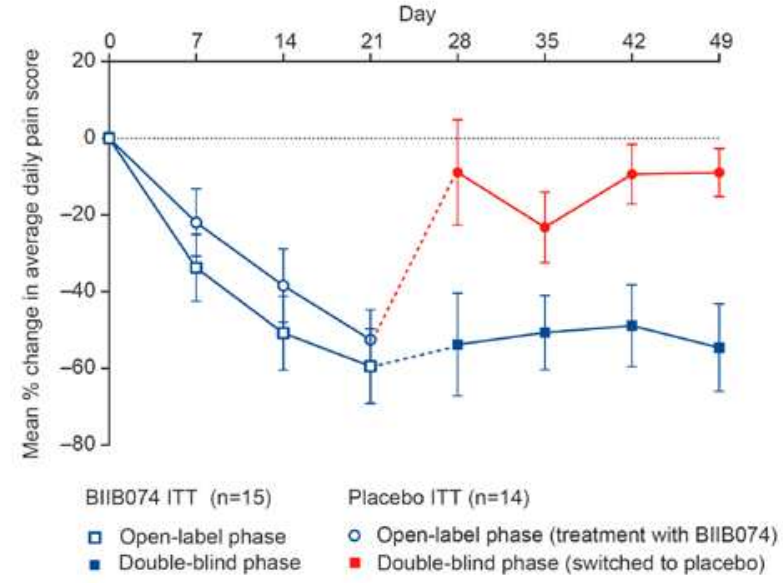

